\title{
Preface
}

The EPL journal has traversed a step-by-step calibrated course over the last one year to serve as a platform for outstanding scholarly works on the global environmental problematique. In view of this the thrust of EPL comprises not only theoretical, procedural, structural and ideational issues of global environmental law and governance but also contemporary sector-specific global regulatory issues.

\section{Challenge of Climate Change}

As a corollary to the above research foci, issue 4 of EPL takes forward the scholarly discourse, from issue 3 , on climate change as one of the common concerns of humankind. In the Anthropocene epoch, climatic changes now carry strong human imprint. There are various manifestations of the challenge of climate change. Issue 3 carried two aspects of adverse impact of climate change in exacerbating sexual and gender-based violence against women as well as decline in the flow of transboundary water resources.

This issue covers a contribution on the adverse effects of "climate change, public insecurity and law" in the wake of growing environmental conflicts involving water resources in a country such as Brazil in the Amazon region. It contends that the immediate and future effects of climate change do have a profound impact on social systems and on the environment. This could become a triggering factor of public insecurity. As such the article suggests that institutions and governments need to address existing effects, and invest in adaptation measures to meet future scientific forecasts on climate change to ensure social stability and the development of a culture of peace.

As a sequel, another contribution on "sea-ice melting, collective Inuit peoples' rights and the human rights discourse" addresses the adverse effects of climatic changes in the Arctic region, where the sea-ice melted faster in 2020 than any time before. It directly threatens the Inuit people's survival, whose livelihood is mainly based on traditional modes of subsistence. Based on international instruments, it has been contended by the authors to recognize the collective rights to communities and people to bridge the human rights gaps in adaptation to the global climatic challenge.

\section{New Concern for Plastics \& Marine Litter}

The emerging global discourse shows that plastic wastes and marine litter has emerged as a new threat to the human environment and the oceans. In the article on "challenge of plastic wastes and litter in oceans" it has been argued that as plastics degrade slowly, its accumulation in the oceans is expected to affect marine ecosystems, ocean fauna and humans. Microplastics threatens to upstage the marine eco-systems. If not addressed urgently, it is feared that by 2050 there would be more plastics than fish in the oceans. The author has called for greater awareness and scientific knowledge about the entire chain as well as innovative approaches to manage plastics wastes. It will necessitate policy, legal and institutional measures for evaluating the trade-offs and gradual shift to the circular plastic economy.

In a related article on "beating plastic pollution", the role of UNEP has been highlighted in bringing visibility to the growing volume of plastic that ends up in landfills, dumps and the environment. It has become a growing cause of concern in developing nations such as India. In the wake of Covid 19 pandemic the enormity of the challenge of plastics has only grown with the use of huge amounts of personal protective equipment (PPEs) and generation of biomedical plastic waste. To address 
the challenge of plastics, the article has called for implementation of the 620 Implementation Framework for Actions on Marine Plastic Litter.

\section{Other Concerns}

The issue covers an off-beat article on "transition to non-lead hunting ammunition in the European Union". The study explains the transition to non-lead ammunition use in the EU through the regional regulatory framework, the EU Regulation 2021/57, that has banned the use of lead gunshot in wetland hunting. The article has highlighted the threats emanating from use of lead ammunition to the health of humans, wildlife, and the environment. It underscores as to how environmental regulatory tool can bring about change in the age-old hunting practices.
Finally, the article on use of 'precautionary principle' in court settlement of civil environmental cases in Indonesia provides a case of the framework for environmental decision-making in the event of scientific uncertainty. Here the application of the 'precautionary approach' in a national legal system provides a good example that could be replicable in other parts of the world.

We hope that these contributions provide a good scholarly churning to address the environmental challenges in respective sectoral areas. They will be of interest to the global scholarly community and the decision-makers alike.

-Bharat H. Desai 